\section{Erklärt «Verschränkung» die Komplementärmedizin?}

Seit gut 2 Jahren deutet sich im Diskurs über das Verständnis des «Komplementären» in der «FORSCHENDEN KOMPLEMENTÄRMEDIZIN UND KLASSISCHEN NATURHEILKUNDE» anhand einer Folge aus wichtigen Originalartikeln zu «Patient-Practitioner-Remedy Entanglement», beginnend mit einer Arbeit von Walach [1] ein neuer Trend an: Demzufolge sei das «Komplementäre» nicht «ergänzend» in Therapieverfahren zu suchen, die «üblicherweise nicht an medizinischen Fakultäten gelehrt werden», wie Eisenberg es definierte. Es liege schon gar nicht in der medizinischen Tradition oder gar der Weltanschauung der beteiligten Personen oder deren jeweiliger Vorstellung von Komplementarität, z.B. in der modernen Physik, begründet, was ebenfalls diskutiert wurde [2,3]. Vielmehr hätten wir es positiv mit neuartigen Wirkmechanismen zu tun, die uns die Quantenphysik eröffne und die offenbar der konventionellen Medizin nicht zugrunde liegen bzw. nicht erforscht oder gezielt genutzt würden.

Zunächst fällt auf, dass die Sprache dieses neuen Diskurses abgehoben ist und dass viele Argumente durch Rückgriffe auf die Physik und ihren Formelapparat verständlicher werden sollen. Folge 8 [4] haben zwei Ärzte meiner Gruppe, beide diplomierte bzw. promovierte Physiker, schlichtweg nicht verstanden. Der Artikel mag in seinen physikalischen Implikationen brillant abgefasst worden sein. Dennoch muss sich der naturwissenschaftlich halbwegs gebildete und theoretisch anspruchsvolle Arzt zwischen unkritischer Hinnahme oder Weiterblättern entscheiden. Immanent fällt Physikern z.B. auf, dass die Wellenfunktion, die das Vielteilchen-Ensemble Mensch beschreiben könnte, ein pures Abstraktum ist, von dem man seriöserweise nicht so sprechen sollte, als könne man es den üblichen Prozeduren für Wellenfunktionen unterwerfen.

Für das Verständnis dieser Wirkmechanismen spielt das Konzept der «Verschränkung» eine zentrale Rolle, eine von vielen möglichen Übersetzungen für «entanglement», was auch Verwicklung, Verwirrung, Gewirr etc. bedeuten kann. Ein brillanter Mathematiker und Autor hat «entanglement» als «the greatest mystery in physics» bezeichnet [5]. Das ist «Verschränkung» zweifellos nicht, dennoch handelt es sich um ein bemerkenswertes Phänomen: Zugrunde liegt ein erstmals 1935 veröffentlichtes berühmtes Gedankenexperiment von Einstein, Podolsky und Rosen, das so genannte «EPR-Paradoxon», das sich vereinfacht gesagt mit einer unvermeidbaren
Koppelung der Information über beliebig weit voneinander entfernt liegende Entitäten, in der ursprünglichen Fassung zwei Photonen, beschäftigt. Durch «Verschränkung» zwischen Therapeut und Patient liessen sich beispielsweise Homöopathie, Fernheilung und viele andere Phänomene verstehen. Ähnliches gelte für Biophotonen, denen sich ein ganzes Heft der Forschenden KomplementÄrmedizin und KLASSISCHEN NATURHEILKUNDE gewidmet hat [6-8]. Der Nachweis der Emission aufeinander abgestimmter, «kohärenter» Photonen durch Lebewesen liess sich zwar inzwischen in mehreren Arbeitsgruppen offenbar bestätigen, eine klinische Bedeutung dieses Phänomens ist jedoch trotz gegenteiliger Bekundung nicht in Sicht. Diese ist auch kaum zu erwarten: höhere Lebewesen müssen für ihre unbestreitbaren Erfolge andere Prinzipien als die fragilen, sehr kurzlebigen der Kohärenz nutzen. Sie sind tendenziell chaotisch angelegt, verfügen jedoch über einige geniale Fähigkeiten, insbesondere ihr kollektiv lernfähiges Gedächtnis, die Replikation der Proteinbiosynthese, um über lange Zeiträume Ordnung, Sinnhaftigkeit, Evolution oder wie immer man es nennen mag, aufzubauen. Diese Entwicklungsfähigkeit geht physikalischen Phänomenen völlig ab - mit Ausnahme der Thermodynamik und ihres Entropieprinzips, das aber statistisch auch eher zu Chaos führt!

Die Physikereliten der ganzen Welt suchen Nachweis und Deutung von Interaktionen, in denen wenige Teilchen in meist extrem kurzen Zeiten und winzigsten Räumen derart puzzeln können, dass man Ordnung, also physikalische Gesetze, nur mithilfe riesiger Rechnerkapazitäten erahnen kann. Schon das Dreiteilchen-Problem, d.h. die Beschreibung dreier miteinander in bekannten Wechselwirkungen stehender Teilchen, bereitet Physikdiplomanden grösstes Kopfzerbrechen. In der Forschung zur Verschränkung ist es kürzlich in einem äusserst pfiffigen Experiment gelungen, diese statt für Einsteins zwei, schon für fünf (!) aufeinander bezogene Photonen nachzuweisen [9]. Auf dieser Betrachtungsebene werden wir nie die Komplexität von Lebewesen mit etwa $10^{28}$ Teilchen aus physikalischen Elementarschritten ableiten können und müssen eine natürlich bei weitem noch nicht verstandene Eigenständigkeit dieser Komplexität anerkennen.

Natürlich muss es weiterhin Grundlagenforschung zu biophysikalischen Grenzeffekten geben. Es wäre jedoch fatal zu glauben, dass sich insbesondere traditionelle europäische wie aussereuropäische Therapieverfahren durch Annahme spezieller, bislang völlig unbekannter Wechselwirkungen wie Verschränkung im quantenmechanischen Sinne zwischen Patient und Therapeut erklären und befördern liessen. Das Gegenteil wird bei konsequenter Verfolgung dieses Weges der Fall sein. 
Darüber hinaus eröffnen vorschnelle Spekulationen über moderne Physik und den kranken Menschen ein Missbrauchspotenzial, das in Form bluffender Analogieschlüsse bereits genutzt wird: Pseudowissenschaftliche Erklärungen der eher obskuren Bioresonanztherapie bedienen sich gerne der Forschungsergebnisse über Biophotonen, ohne dass sich einer deren Vertreter davon distanziert hätte.

Die Homöopathie konfrontiert uns mit der provokantesten Hypothese eines nicht verstandenen Wirkmechanismus in der Komplementärmedizin. Viele ihrer Anhänger haben Erklärungsversuche beigesteuert, denen zufolge ein Medikament auch in Abwesenheit einer wirksamen Substanz Effekte erzeugen kann. Auch gut 20 Jahre nach den ersten Spekulationen der Wiener Schule um Gutsch über einen physikalisch nachvollziehbaren Informationsübertrag auf das Lösungsmittel Wasser durch die Verschüttelung, die auf seiner sehr komplizierten so genannten Nahordnung basieren könnten, sind wir hier allenfalls Millimeter weiter gekommen. Witt et al. [10] beschreiben solche möglicherweise nachweisbaren, aber nicht interpretierbaren Spuren. In der Frage der Bewertung des therapeutischen Nutzens der Homöopathie sind wir trotz ihrer weiten Verbreitung nicht weitergekommen.

Wissenschaftshistorisch finden sich interessant zu vergleichende Situationen, z.B. der «ganzheitliche» Ansatz, der etwa ab 1900 zunächst in der Biologie, der Psychologie, später auch der Neurologie und letztlich der klinischen Medizin insgesamt, zeitweilig auch in der Naturheilkunde, äusserst virulent auftrat [11]. Seine Vertreter liefen immer wieder Sturm gegen mechanistische, fragmentierte Sichtweisen auf Lebewesen durch die Einzeldisziplinen und hofften auf grundlegende Ordnung - ein äusserst lebhafter, letztlich aber erfolgloser Diskurs. In der Physik hat es ähnliche Diskussionen gegeben. Auch hier hat es nicht an «ganzheitlichen» Vertretern gefehlt, die im vermeintlichen Chaos verlässliche Ordnung herstellen wollten. Einer der renommiertesten Vertreter war Werner Heisenberg. Dass seine berühmte Weltformel nach Jahrzehnten des Streits nicht akzeptiert, aber auch nicht sicher widerlegt wurde, kennzeichnet die Tragik dieses Anspruchs.

Wo liegen unsere Entwicklungsmöglichkeiten? Die Annahme, dass sich die Komplementärmedizin bevorzugt neuer, tendenziell unanschaulicher und derzeit schwer nachweisbarer physikalischer bzw. physikalisch-chemischer Wirkmechanismen bediene, kann natürlich zutreffen, Stück für Stück belegt werden und ihr letztlich über die Grundlagenforschung zum Durchbruch verhelfen. Für eine solche Entwicklungsmöglichkeit spricht meines Erachtens nichts. Für die Klassische Naturheilkunde wäre die konsequente Verfolgung dieses Ansatzes abenteuerlich und selbstzerstörerisch. Ihre Vertreter müssten bei einer Konzentrierung auf solche Forschungsansätze andere Diskussions- und Arbeitszusammenhänge aufsuchen. Umgekehrt ist natürlich eine klinische Forschung, die nur auf den Autobahnen RCT, GCP, EBM, Metaanalyse und wie immer die Schlagwörter lauten mögen, welche die zunehmende Bürokratisierung der medizinischen Forschung charakterisieren, entlangbrummt, zur Langweiligkeit verurteilt. Doch wo liegen neue Chancen, neue Ideen, neue Bündnispartner? Sicherlich nicht in der Quantenmechanik, sondern in den Kultur- und Geschichtswissenschaften, der Anthropologie, einer ernsthaft um das oft verzweifelte Subjekt bemühten klinischen Psychologie und Psychotherapie, den Kreativ- und Körpertherapien, der Meditation und und und! Gerade heute, wo selbst die konventionelle Medizin im Diagnose- und Therapieprozess das lange sträflich missachtete Subjekt wieder zu entdecken beginnt, wäre es fatal, wenn eine unkonventionelle Medizin dessen Therapiehoffnungen in eine kaum verstehbare Quantenphysik zu pressen versuchte, anstatt seine Äusserungen zu sehen und zu respektieren: die Orientierung kulturell und historisch verankerter erkrankter bzw. Prävention suchender Menschen zunächst an den Heilmitteln ihres eigenen, darüber hinaus auch anderer Kulturkreise, die keine Beschränkung darstellt, sondern insbesondere Ich-Stärke, Zufriedenheit und Compliance fördert. Trotz ihrer führenden Stellung in der konservativen Therapie über gut ein Jahrhundert und ihres immer noch wachsenden Machtanspruchs ist die breite Verdrossenheit über die Pillenmedizin jedem sensiblen Arzt täglich spürbar, das Verlangen nach Wahrnehmung, Akzeptanz, Identifizierung mit dem Heilmittel, das eine chemisch definierte Substanz auch im Computerzeitalter nie herstellen kann. Was für Riesenchancen!

R. Stange, Berlin

1 Walach H: Entanglement model of homeopathy as an example of generalized entanglement predicted by weak quantum theory. Forsch Komplementärmed Klass Naturheilkd 2003;10:192-200.

2 Stange R: Noch einmal - das Komplementäre in der Medizin. Forsch Komplementärmed 2000;7:117-118.

3 Meyer-Abich KM: Naturheilkunde oder Komplementärmedizin. Forsch Komplementärmed 1999;6:293.

4 Milgrom LR: Patient-Practitioner-Remedy (PPR) entanglement, Part 8: 'Laserlike' action of the homeopathic therapeutic encounter as predicted by a gyroscopic metaphor for the vital force. Forsch Komplementärmed Klass Naturheilkd 2005;12:206-213.

5 Aczel AD: Entanglement: The Greatest Mystery in Physics. San Francisco, Wiley, 2002.

6 Schwabl H: 100 Jahre nach Einsteins Wunderjahr: Die elektromagnetische Wechselwirkung als Stiefkind der CAM-Forschung? Forsch Komplementärmed Klass Naturheilkd 2005;12:72-73.

7 Walach H: Licht: Zwischen Materie und Energie. Forsch Komplementärmed Klass Naturheilkd 2005;12:74-75.

8 Van Wijk R, Van Wijk EPA: An introduction to human biophoton emission. Forsch Komplementärmed Klass Naturheilkd 2005;12:77-83.

9 Zhao Z, Chen YA, Zahng AN, Yang T, Briegel HJ, Pan JW: Experimental demonstration of five-photon entanglement and open-destination teleportation Nature 2004;430:54-58.

10 Witt C, Lüdtke R, Weisshuhn TER, Willich SN: High homeopathic potencies are different from potentized solvent when investigated with the REDEM technology. Forsch Komplementärmed Klass Naturheilkd 2005;12:6-13.

11 Harrington A: Die Suche nach Ganzheit. Reinbek, Rowohlt, 2002.

\section{Antwort des Hauptherausgebers}

Rainer Stange weist darauf hin, dass in letzter Zeit vermehrt Arbeiten zur «quantenmechanischen Betrachtung in der Komplementärmedizin» erschienen sind. Er beklagt diese 
Entwicklung als fehlgeleitet, da sie dem Praktiker nicht wirklich helfe, die Öffentlichkeit in eine pseudowissenschaftliche und vermeintliche, theoretische Sicherheit führe und damit insgesamt Ruf und Ansehen der Komplementärmedizin schade.

Ich möchte Rainer Stange sein Empfinden und seine Erfahrung nicht absprechen und gehe davon aus, dass seine Reaktion typisch für viele Praktiker und damit auch für viele Leser der Forschenden KomplementäRMEDIZIN sein könnte. Dies wundert mich nicht. Wann immer neue theoretische Modelle eingeführt und alte Denkgewohnheiten in Frage gestellt werden, stellen sich zunächst Unsicherheit, Unverständnis und Widerstand ein. Ich möchte an dieser Stelle die Gründe, die für die neue Denkweise sprechen, nicht wiederholen; dies ist in den entsprechenden Originalarbeiten [1-5] geschehen. Einen Punkt gilt es allerdings hervorzuheben: Gehen wir einmal davon aus, Stange hat Recht. Gehen wir davon aus, dass die herkömmliche klassische Theoriebildung vollauf genügt, um die Komplementärmedizin zu verstehen und um sie in den Corpus des Gesamtwissens einzubetten. Dann bleiben im Wesentlichen zwei Möglichkeiten offen: Erstens, die Komplementärmedizin belegt mit einer Serie von methodisch sauberen und pragmatisch erfolgreichen Studien die Spezifität ihrer Effekte. Sie folgt den Postulaten und Dogmen der evidenzbasierten Medizin und führt Serien von randomisierten, idealerweise verblindeten Studien durch, die am Schluss in einer Metaanalyse zweifelsfrei belegen, dass komplementärmedizinische Verfahren kausale und stabile Effekte aufweisen. Dies ist bislang nur in wenigen Fällen eine Erfolg versprechende Strategie gewesen und hat nach meinem Dafürhalten noch kaum Kritiker von der Brauchbarkeit der Komplementärmedizin überzeugt. Die Tendenz, die abzusehen ist, ist eher die, dass das Verfolgen dieses Pfades dazu führt, dass komplementärmedizinische Verfahren als gleichwertig zu Plazebo angesehen werden. Dann bleibt dieser Denkweise nur der zweite Ausweg: zu argumentieren, dass Plazeboeffekte Selbstheilungseffekte sind und als solche wertvoll für unser Gesundheitswesen. Dann wird es nötig sein, diese komplexen Selbstheilungsprozesse genauer zu untersuchen und zu zeigen, dass sie trotz des Fehlens eines unspezifischen Elements bedeutsam und für die Öffentlichkeit finanziell tragbar sind. Die Komplementärmedizin befindet sich an dieser Stelle in guter Gesellschaft: psychotherapeutische Verfahren - und darauf weist Stange ja hin - haben wohl ähnliche Probleme und bedienen sich einer ähnlichen Argumentationsweise.

Die Motivation, einen anderen Weg einzuschlagen, ist eine zweifache: Erstens legt die Phänomenologie nahe, dass manche komplementärmedizinischen Verfahren, etwa die Homöopathie, durchaus spezifische Effekte haben können, dass diese aber in keiner Weise kausal stabil sind. Vielmehr sind sie sehr empfindlich, ja fast volatil, und in einem kausalen Testmodell meistens instabil. Daraus würde man klassischerweise schliessen, dass Homöopathie eben Plazebo sei. Genau diese Schlussfolgerung scheint mir zu kurz gegriffen, und deswegen haben sich, übrigens unabhängig voneinander, unterschiedliche Bestrebungen ergeben, ein neues Denkmodell zu etablieren. - Die zweite Motivation für die Einführung eines neuen Modells ist geisteswissenschaftlicher, ja vielleicht sogar kulturwissenschaftlicher Natur: Seit etwa 100 Jahren bedient sich die Physik einer Denkfigur, die sich von der klassischen Denkweise fundamental unterscheidet. Diese Denkfigur hat zu sehr tief greifenden, ja sogar radikalen Neuinterpretationen der Welt Anlass gegeben. Unter anderem dazu, die Welt als ein in ihrer Tiefenstruktur verbundenes System zu verstehen. Die Extrapolation der klassischen Denkweise auf alle möglichen menschlichen Zusammenhänge, insbesondere auf die Heilkunst und auf zwischenmenschliche Interaktion, scheint mir persönlich ein schlimmer Anachronismus zu sein. Im besten Falle erreichen wir dadurch Annäherungen, aber keinesfalls präzise Beschreibungen.

Auf ein wichtiges Missverständnis und eine Fehlrezeption Stanges möchte ich den Leser hinweisen: alle publizierten theoretischen Ansätze haben gemeinsam, dass sie die Quantenmechanik nicht einfach naiv in die Aussenwelt extrapolieren. Es geht darum, die Struktur des quantenmechanischen Denkens beizubehalten, nicht darum, so zu tun, als wären alle Elemente unserer Welt einfach quantenmechanische Objekte. Dies ist ein subtiler, aber wichtiger Unterschied. Wenn dieser einmal verstanden ist, verschwinden eigentlich alle Probleme, von denen Stange spricht. Dann bleibt eigentlich nur noch ein Problem: diese Denkweise nämlich wirklich zu verstehen. Dies ist nicht einfach und geht auch nicht von heute auf morgen. Stanges Brief zeigt dies deutlich. Meine persönliche Meinung ist, dass es sowohl notwendig als auch lohnend ist.

H. Walach, Northampton

\section{Literatur}

1 Schmid GB: Much ado about entanglement: A novel approach to test nonloca communication via violation of 'Local Realism'. Forsch Komplementärmed Klass Naturheilkd 2005;12:214-222.

2 Hyland ME: A brief guide to extended network entanglement theory as a theory of healing and its empirical predictions. Forsch Komplementärmed Klass Naturheilkd 2003;10:201-206.

3 Milgrom LR: Patient-practitioner-remedy (PPR) entanglement. Part 8: 'Laserlike' action of the homeopathic therapeutic encounter as predicted by a gyroscopic metaphor for the vital force. Forsch Komplementärmed Klass Naturheilkd 2005;12:206-213

4 Walach H: Entanglement model of homeopathy as an example of generalized entanglement predicted by weak quantum theory. Forsch Komplementärmed Klass Naturheilkd 2003;10:192-200.

5 Weingärtner O: Über die wissenschaftliche Bearbeitbarkeit der Identifikation eines «arzneilichen Gehalts» von Hochpotenzen. Forsch Komplementärmed Klass Naturheilkd 2002;9:229-233. 